\title{
Non-abelian superconducting pumps
}

\author{
Valentina Brosco, ${ }^{1,2}$ Rosario Fazio, ${ }^{3,4}$ F.W.J. Hekking, ${ }^{5}$ and Alain Joye ${ }^{5,6}$ \\ ${ }^{1}$ Institut für Theoretische Festkörperphysik and DFG Center for Functional Nanostructures (CFN), \\ Universität Karlsruhe, 76128 Karlsruhe, Germany \\ ${ }^{2}$ NEST-CNR-INFM $\&$ Dipartimento di Fisica, Universitá di Pisa, largo E. Fermi, I-56100 Pisa, Italy \\ ${ }^{3}$ International School for Advanced Studies (SISSA) via Beirut 2-4, I-34014, Trieste - Italy \\ ${ }^{4}$ NEST-CNR-INFM \&5 Scuola Normale Superiore, piazza dei Cavalieri 7, I-56126 Pisa, Italy \\ ${ }^{5}$ LPMMC, CNRS \& Université Joseph Fourier, BP 166, 38042 Grenoble CEDEX 9, France \\ ${ }^{6}$ Institut Fourier, BP 74, 38402 Saint-Martin d'Hères, France
}

\begin{abstract}
Cooper pair pumping is a coherent process. We derive a general expression for the adiabatic pumped charge in superconducting nanocircuits in the presence of level degeneracy and relate it to non-Abelian holonomies of Wilczek and Zee. We discuss an experimental system where the non-Abelian structure of the adiabatic evolution manifests in the pumped charge.
\end{abstract}

PACS numbers:

If the Hamiltonian of a quantum system depends adiabatically and cyclically on time via some external parameters the wavefunction, for the system initially prepared in an energy eigenstate, after a cycle returns back to its initial state up to a phase, that in addition to the dynamical contribution, has a component of geometric nature [1]. It depends only on the shape of the path covered in the parameters space and on the structure of the Hilbert space of the quantum system. If the adiabatic evolution takes place in a degenerate energy eigenspace the cyclic evolution leads to a superposition of the degenerate eigenstates and the geometric transformation acquires a non-Abelian structure [2]. Simon [3] and Wilczek and Zee [2] showed that this mapping plays the role of the holonomy of the gauge theories. Holonomies naturally emerge in the description of the dynamics of simple quantum systems due to the parallel transport dictated by the Schroedinger equation $[4,5]$ which constraints the overlap between the wavefunctions of the system at successive times to be real and positive. The holonomy group is the group of the transformations generated by the parallel transport along closed paths on the parameter space.

Geometric effects appear naturally in adiabatic quantum pumping: in a mesoscopic conductor a dc charge current can be obtained, in the absence of applied voltages, by cycling in time two parameters which characterize the system [6]. In the scattering approach to transport the pumped charge per cycle can be expressed in terms of derivatives of the scattering amplitudes with respect to the pumping parameters [7], the Brouwer formula [8]. Its relation to geometric phases has been elucidated in Refs. $[9,10]$. If only superconducting leads are present pumping is due to the adiabatic transport of Cooper pairs. Besides the dependence of the pumped charge on the cycle, in this case there is a dependence on the superconducting phase difference(s) (the overall process is coherent). Cooper pair pumping has been studied both in the limit of transparent interfaces [11, 12] and in the Coulomb Blockade regime [13-15]. A connection between Berry phase and pumped charge has been established also in this case $[11,16,17]$ thus opening the possibility to detect geometric phases in superconducting circuits $[17,18]$. An experiment of this kind has been successfully performed recently in Ref. [19] thus paving the way to holonomic quantum computation $[20,21]$ with superconducting nanodevices.

In this Letter we study Cooper pair pumping in superconducting circuits in the regime of Coulomb blockade. The new feature we consider here is the possibility to pump in a degenerate subspace. We derive an expression for the pumped charge in the presence of a degenerate spectrum and relate it to the non-Abelian connection of Wilczek and Zee. We propose a superconducting network where this relation can be tested and discuss two clear signatures of non-abelian holonomies. First, under appropriate conditions, the pumped charge per cycle is quantized. Second the pumped charge depends both on the cycle and on the point where the cycle starts. If tested experimentally this would be a clear proof of the non-Abelian nature of pumping.

The possibility to generate non-abelian holonomies in superconducting circuits has been studied previously [22]. Ref.[22] dealt with the problem of implementing a holonomic quantum computer with Josephson circuits. In that work the authors show that a cyclic adiabatic change of parameters in a closed system may lead to a final state having a different charge distribution than the initial one. In that context the word "charge pumping" was used to describe such process even if the charge cannot be really pumped in or out the system. In the present work we study charge transport between two external reservoirs connected to the circuit via Josephson junctions, the word "pumping" has thus a different meaning. In order to keep the presentation transparent we discuss an idealized situation, in the conclusions we discuss the various problems that may occur in experiments. The Cooper pair pump consists of a Josephson network connected through Josephson junctions to two superconduct- 
ing leads. An example (at this stage the discussion is general) is presented in Fig. 1. The two superconducting electrodes are kept at a finite phase difference $\varphi=$ $\varphi_{L}-\varphi_{R}$ where $\varphi_{R / L}$ is the phase of the superconducting order parameter of the right/left lead. The Cooper pair pump is operated by changing adiabatically in time some external parameters such as gate voltages or magnetic fluxes. We will label this set of external parameters by the vector $\vec{\lambda}(t)=\left\{V_{g i}(t), \Phi_{i}(t)\right\}$. The Hamiltonian of the pump depends on the superconducting phase of each island of the network $\varphi_{i}(i=1, \ldots N)$, on its conjugate, the charge on each island $n_{i}$, on the phase difference across the pump, $\varphi$, and on all the external parameters: $H(t)=H\left[\varphi_{1}, \ldots, \varphi_{N} ; n_{1}, \ldots, n_{N} ; \vec{\lambda}(t), \varphi\right]$. The state of the system is denoted by $|\Psi(t)\rangle$ and $|\Psi(t)\rangle=|\Psi[t, \vec{\lambda}(t), \varphi]\rangle$. By changing the control parameters in time, a charge $Q^{(t r)}$ will be transferred in the circuit. The transferred charge after a period $T$ can be obtained by integrating the charge imbalance between the outer capacitors that connect the network to the leads

$$
Q^{(t r)}=-2 i e \int_{0}^{T} \partial_{t^{\prime}}\left[\left\langle\Psi\left(t^{\prime}\right)\left|\partial_{\varphi}\right| \Psi\left(t^{\prime}\right)\right\rangle\right] d t^{\prime}
$$

This definition of the transferred charge may be derived from the time integral of the current operator, $Q^{(t r)}=$ $-\frac{2 e}{\hbar} \int_{0}^{T}\left\langle\Psi\left(t^{\prime}\right)\left|\left(\partial_{\varphi} H(t)\right)\right| \Psi\left(t^{\prime}\right)\right\rangle d t^{\prime}$, and the Schrödinger equation. In the definition of the transferred charge $\partial_{\varphi}$ is not a quantum operator but a simple derivative respect to a classical parameter (the phase difference between the two electrodes). In this Letter we generalize the results obtained so far relating Cooper pair pumping to geometric phases allowing the spectrum of $H(t)$ to be degenerate. We assume that for all $\vec{\lambda}$ in the parameter space a degenerate energy eigenspace $\mathcal{H}_{n}(\vec{\lambda})$ exists of constant dimension, $D_{n}$, corresponding to the eigenvalue $E_{n}(\vec{\lambda})$. The control parameters are varied in time adiabatically, i.e. $\vec{\lambda}=\vec{\lambda}(t / T)$. It is convenient to introduce for all $t \in[0, T]$ a basis, $\left\{\psi_{n \alpha}(t)\right\} \alpha \in\left[1 . . D_{n}\right]$, of the degenerate subspace, $\mathcal{H}_{n}(\vec{\lambda})$, formed by the instantaneous eigenstates of the hamiltonian, $H(t): H(t)\left|\psi_{k \alpha}(t)\right\rangle=$ $E_{k}(t)\left|\psi_{k \alpha}(t)\right\rangle$. As discussed by Wilczek and Zee [2], if initially the state of the system is in one of the degenerate eigenstates $|\Psi(t=0)\rangle=\left|\psi_{n \nu}[\vec{\lambda}(0)]\right\rangle \in \mathcal{H}_{n}(\vec{\lambda}(0))$, then after a cyclic evolution

$$
|\Psi(T)\rangle=\left[U_{n}(T)\right]_{\alpha \nu}\left|\psi_{n \alpha}(\vec{\lambda}(0))\right\rangle+\mathcal{O}(1 / T) .
$$

The $D_{n} \times D_{n}$ operator $U_{n}(t)$ can be written as

$$
U_{n}(T)=e^{-\frac{i}{\hbar} \int_{0}^{T} E_{n}(t)} \mathcal{T} e^{\left[-\int_{0}^{T} \Gamma_{n}(t) d t\right]} .
$$

Here, $\mathcal{T}$ denotes the time-ordering and the connection $\Gamma_{n}(t)$ is given by $\left[\Gamma_{n}(t)\right]_{\alpha \beta}=\left\langle\psi_{n \alpha}(t) \mid \dot{\psi}_{n \beta}(t)\right\rangle$. The relation between the transferred charge and the non-Abelian holonomy can be obtained by substituting (2) in (1) [23]. We assume that the system is prepared at $t=0$ in a linear superposition of the degenerate eigenstates, $\left|\Psi_{i n}\right\rangle=\sum_{\gamma} c_{\gamma}\left|\psi_{n \gamma}[\vec{\lambda}(0)]\right\rangle ;$ during a cycle of duration $T$ the total transferred charge then reads

$$
Q^{(t r)}=\sum_{\gamma \gamma^{\prime}} c_{\gamma}^{*} c_{\gamma^{\prime}} \hat{Q}_{\gamma \gamma^{\prime}}^{n}
$$

where the charge matrix $\hat{Q}_{\gamma \gamma^{\prime}}^{n}$ is given by

$$
\hat{Q}_{\gamma \gamma^{\prime}}^{n}=-\frac{2 e}{\hbar} \int_{0}^{T}\left\{\partial_{\varphi} E_{n} \delta_{\gamma \gamma^{\prime}}-i \hbar \sum_{\alpha \beta}\left[\left[U_{n}^{\dagger}\right]_{\gamma \alpha}\left(\partial_{\varphi}\left[\Gamma_{n}\right]_{\alpha \beta}\right)\left[U_{n}\right]_{\beta \nu}-\partial_{t}\left(\left[U_{n}^{\dagger}\right]_{\nu \alpha}\left\langle\psi_{n \alpha}\left|\partial_{\varphi}\right| \psi_{n \beta}\right\rangle\left[U_{n}\right]_{\beta \gamma^{\prime}}\right)\right]\right\} d t+\mathcal{O}(1 / T)
$$

The first term in the r.h.s. is the supercurrent contribution to the transferred charge. The second and third terms are of geometrical nature and describe pumping. If the pumping occurs through a non-degenerate level, $D_{n}=1$, we recover the abelian result $[16,17,24]$. The measurement of non abelian holonomies is a non-trivial task, in particular the non commutativity of the theory has revealed not easy to detect [5]. Non-abelian contributions to pumping introduce qualitatively new effects that can be verified experimentally. We will highlight these aspects by analyzing a specific case that can be realized experimentally.

A possible experimental realization of a non-abelian pump is shown in Fig.1. It is a three island pump with four symmetric Josephson SQUID loops: two inner loops with capacitances $C_{1}$ and $C_{2}$ and two outer loops with capacitances $C_{L}$ and $C_{R}$. The outer loops connect the network to the superconducting electrodes which are kept at a constant phase difference $\varphi$ (from now on for simplicity we fix $\varphi_{L}=\varphi$ and $\left.\varphi_{R}=0\right)$. The charging configuration of the system can be controlled externally modulating three gate voltages, $V_{u}, V_{g 1}$ and $V_{g 2}$ connected to the islands via the respective gate capacitances, $C_{u}, C_{g 1}$ and $C_{g 2}$. The effective Josephson couplings, $J_{L}, J_{R}, J_{1}, J_{2}$, can be tuned independently varying the magnetic fluxes through each loop. All the Josephson coupling energies are much smaller than the charging energy of the system, $E_{C}$. The charge states are indicated as $\left|n_{u}, n_{1}, n_{2}\right\rangle$, 
$n_{i}$ being the excess charge on the $i$-th island in units of $2 e$. The realization of the degenerate subspace requires additional constraints on the gate voltages. We take the gate capacitances to be small compared to the Josephson capacitances, i.e. $C_{u} \sim C_{g i} \ll C_{i} \sim C_{I}$ with $i=1,2$ and $I=L, R$, and we assume that the gate charge $n_{g u}=C_{u} V_{g u} / 2 e$ is in the range $\frac{1}{2}<n_{g u}<\frac{3}{2}+\frac{C_{1}+C_{2}}{C_{L}+C_{R}}$ while the two gate charge $n_{g i}=C_{g i} V_{g i} / 2 e$ satisfy the condition $n_{g i}=\frac{1}{2}\left(1+\frac{C_{i}}{C_{T}}\left(2 n_{g u}-1\right)\right)$ with $i=1,2$ and $C_{T}=C_{1}+C_{2}+C_{L}+C_{R}$. Under these conditions only four charge states are relevant to the dynamics at low temperatures: $|0,0,0\rangle,|0,1,0\rangle,|0,0,1\rangle,|1,0,0\rangle$ (all other charge states are at a much higher energy $\left.\sim E_{C}\right)$. Moreover the charge states $|0,0,0\rangle,|0,1,0\rangle,|0,0,1\rangle$ are degenerate while the charge state $|1,0,0\rangle$, corresponding to the configuration in which there is one excess charge on the island $U$, has higher energy. In this restricted Hilbert space the effective hamiltonian of the pump can be written as

$$
\begin{aligned}
H & =E_{u}|1,0,0\rangle\langle 1,0,0|+\left[J_{\text {eff }}(\varphi)|1,0,0\rangle\langle 0,0,0|\right. \\
& \left.+J_{1}|1,0,0\rangle\left\langle 0,1,0\left|+J_{2}\right| 1,0,0\right\rangle\langle 0,0,1|+\text { h.c. }\right](6)
\end{aligned}
$$

where $J_{\text {eff }}(\varphi)=\left(J_{L} e^{i \varphi}+J_{R}\right)$. We set to zero the electrostatic energy of the degenerate charge configurations and we denoted with $E_{u}$ the charging energy of the state $|1,0,0\rangle, E_{u}=\frac{2 e^{2} C_{1}\left(2 n_{g 1}-1\right)}{\left(C_{L}+C_{R}\right)^{2}}$. Given the capacitances, our choice of the gate voltages guarantees that the charging hamiltonian is symmetric under the simultaneous exchange of the three charge states and of the three couplings $J_{\text {eff }}, J_{1}$ and $J_{2}$. This fact leads to a two dimensional degenerate subspace for any value of the couplings. Hamiltonian (6) has been discussed previously in the context of adiabatic passage techniques in a quantum optics [25] and superconducting nanocircuits [27] and for holonomic quantum computation as demonstrated in [26]. The crucial point here is that Eq. (6) arises from a Josephson network in the presence of superconducting electrodes. This is why we are able to relate pumping to holonomies. The hamiltonian has three distinct eigenvalues: $E_{0}=$ $1 / 2\left[E_{u}-\left(E_{u}^{2}+4\left(J_{0}^{2}+J_{1}^{2}+J_{2}^{2}\right)\right)^{1 / 2}\right], E_{1}=0$ and $E_{2}=$ $1 / 2\left[E_{u}+\left(E_{u}^{2}+4\left(J_{0}^{2}+J_{1}^{2}+J_{2}^{2}\right)\right)^{1 / 2}\right]$. In the previous definitions we set $J_{0}=\left(J_{L}^{2}+J_{R}^{2}+2 J_{L} J_{R} \cos \varphi\right)^{1 / 2}$. The eigenvalue $E_{1}$ remains zero and it is doubly degenerate for any value of the Josephson couplings not all zero. An orthonormal basis for the two-dimensional degenerate subspace corresponding to the eigenvalue $E_{1}$ is given by : $\left|\psi_{11}\right\rangle=N_{11}\left(J_{2}|0,1,0\rangle-J_{1}|0,0,1\rangle\right)$ and $\left|\psi_{12}\right\rangle=$ $N_{12}\left[\left(J_{1}^{2}+J_{2}^{2}\right)|0,0,0\rangle-J_{\text {eff }}(\varphi)\left(J_{1}|0,1,0\rangle+J_{2}|0,0,1\rangle\right)\right]$ where $N_{11}$ and $N_{12}$ are normalization factors.

In the following we will show that: (i) The pumped charge may be quantized; (ii) By composing two cycles in the parameter space, the pumped charge depends on the order in which the two cycles are followed. (iii) Consider-

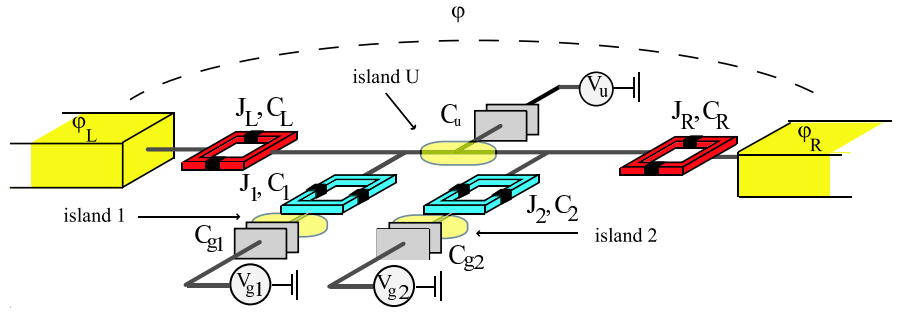

Figure 1: Non-abelian superconducting pump. The gate voltages are kept fixed during the cycle, their values are chosen in order to have a doubly degenerate spectrum. The only pumping parameters are the fluxes through the four loops.

ing the pumped charge as a function of time, $Q=Q(t)$, the period of $Q(t)$ may be a multiple of the period of the parameters cycle. The second and the third point are due to the non-abelian character of the adiabatic evolution.

(i) We first discuss the quantization of the pumped charge. To this end the cycle can be divided in three steps. First, we prepare the system in the charge state $|0,0,0\rangle$ and we set all the couplings to zero except for $J_{2}$ which is kept constant and positive during the whole cycle. For these initial values of the couplings the two degenerate eigenstates are also charge eigenstates: $\left|\psi_{11}(t=0)\right\rangle=|0,1,0\rangle,\left|\psi_{12}(t=0)\right\rangle=|0,0,0\rangle$; so the system is initially in the eigenstate $\left|\psi_{12}\right\rangle$. Second, we perform a $\pi / 2$ rotation in the degenerate subspace by manipulating adiabatically and cyclically $J_{L}$ and $J_{1}$ and keeping $J_{R}$ to zero. In this phase one charge enters the pump from the left reservoir and the state vector of the system undergoes the transformation: $|0,0,0\rangle \rightarrow e^{i \varphi}|0,1,0\rangle$. Third, we let the charge out of the circuit with another $\pi / 2$ rotation in the degenerate subspace. During this third phase we manipulate $J_{R}$ and $J_{1}$ and we keep $J_{L}$ to zero. The state of the system undergoes the transformation $e^{i \varphi}|0,1,0\rangle \rightarrow e^{i \varphi}|0,0,0\rangle$. How is it possible to realize the $\pi / 2$ rotations? One can show that by manipulating adiabatically $J_{1}, J_{2}$ and $J_{L}$ or $J_{1}, J_{2}$ and $J_{R}$ and
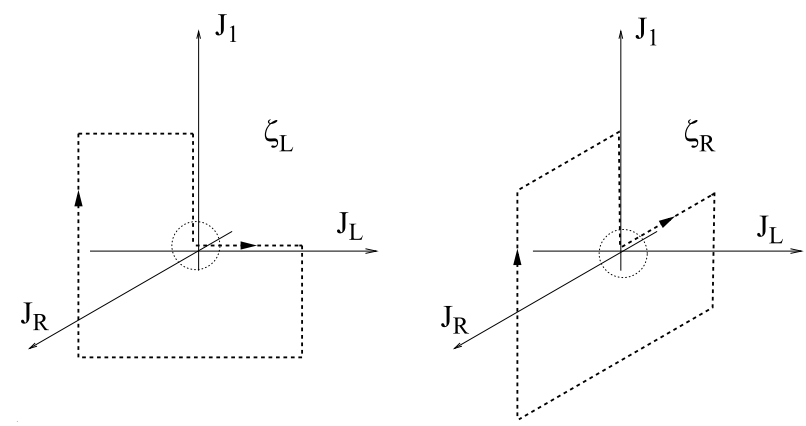

Figure 2: By varying the Josephson couplings through the cycles $\zeta_{L}$ (left) and then $\zeta_{R}$ (right) one Cooper pair is transferred through the pump. 

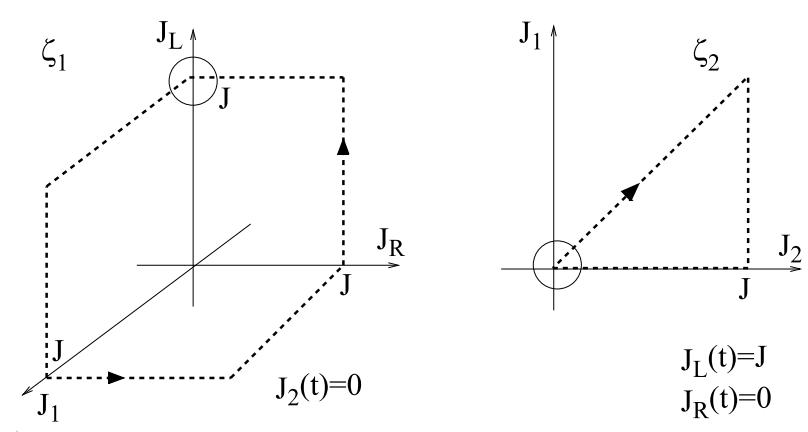

Figure 3: Non commuting cycles in the parameters space. Along $\zeta_{1}, J_{2}$ is set to zero while along $\zeta_{2}$, both $J_{L}$ and $J_{R}$ are fixed. The circles indicate the initial points of each cycle.

keeping respectively $J_{R}$ or $J_{L}$ zero the holonomy reads

$$
U_{L / R}^{(\zeta)}=\left(\begin{array}{cc}
\cos \Omega_{\zeta} & e^{i \varphi_{L / R}} \sin \Omega_{\zeta} \\
-e^{-i \varphi_{L / R}} \sin \Omega_{\zeta} & \cos \Omega_{\zeta}
\end{array}\right)
$$

where $\Omega_{\zeta}=\oint_{\zeta}\left\langle\psi_{11} \mid \dot{\psi}_{12}\right\rangle$. The required rotations are realized whenever $\Omega_{\zeta}=\pi / 2$, for example by means of the cycles $\zeta_{L}$ and $\zeta_{R}$ shown in Fig. 2. By substituting the evolution $U$, the connection $\Gamma$ and the matrix elements of $\left\langle\psi_{n \alpha}\left|\partial_{\varphi}\right| \psi_{n \beta}\right\rangle$ for the cycles of Fig. 2 in Eq. (5) we find $Q^{(t r)}=-2 e$. For the present cycle, the charge pumped starting from the eigenstate $\left|\psi_{11}\right\rangle$ has opposite sign to the charge pumped starting from the eigenstate $\left|\psi_{12}\right\rangle$. (ii) The non-abelian nature of the evolution has measurable effects on the pumped charge. We consider the cycles depicted in Fig. 3 and determine the pumped charge in the two cases in which the pumping cycle is either performed by first going over $\zeta_{1}$ and then $\zeta_{2}$ or in the reverse order. In the abelian case considered in Refs. $[14,16,17]$ the two situations are equivalent. In our case the order matters, i.e. there are examples where $Q_{\zeta_{1} \zeta_{2}}^{(t r)} \neq Q_{\zeta_{2} \zeta_{1}}^{(t r)}$. A specific example is provided by the pumping cycle obtained by performing the two loops presented in Fig. 2. Assuming that the initial state is the state $|0,1,0\rangle$, the pumped charge in the two cases is $Q_{\zeta_{1} \zeta_{2}}^{(t r)}=\frac{e}{2}$ and $Q_{\zeta_{2} \zeta_{1}}^{(t r)}=e$. The pumped charge differs in the two cases.

(iii) Another manifestation of the non-Abelian nature of pumping is that after a cyclic evolution of the external parameters the state does not necessarily go to the initial state (see Eq. (2)). One can therefore design paths in parameter space such that after $N$ cycles the system returns, up to a phase, to its initial state. In this situation the pumped charge per cycle will not be constant in each cycle but it will have a period which is $N T, T$ being the period of the elementary cycle. In fact, assume that a certain cycle $\bar{\zeta}$ is performed consecutively $\mathrm{N}$ times starting with the system in the state $\left|\Psi_{i n}\right\rangle$ defined before. At the beginning of the $\mathrm{N}$-th cycle the state will be $\left(U_{\bar{\zeta}}\right)^{N-1}\left|\Psi_{i n}\right\rangle$ then, as one can easily show using Eq. (4), the total transferred charge will be given by: $Q_{\text {Nth cycle }}^{t r}=\sum_{\gamma \gamma^{\prime}} c_{\gamma}^{*} c_{\gamma^{\prime}}\left[\left(U_{\bar{\zeta}}^{\dagger}\right)^{N-1} \hat{Q}^{n}\left(U_{\bar{\zeta}}\right)^{N-1}\right]_{\gamma \gamma^{\prime}}$.

Eventually, when $U_{\bar{\zeta}}^{N-1}$ is proportional to the identity operator, the transferred charge will be periodic with period $N T$.

The situation we discussed so far is ideal. Several important issues have to be considered in a realistic situation. First of all, it would be desirable to pump through the ground state; here the degenerate subspace is the first excited level, which is sensitive to relaxation effects. We do not think this is a serious problem, though: we showed how the state can be accessed and moreover the real bottleneck is the decoherence time and not the relaxation time, which typically is much longer. Indeed, the effect of decoherence on coherent pumping appears to be a more fundamental issue since the presence of an external bath may, in addition to relaxation, lift part of the degeneracy which is crucial for non-Abelian pumping. In addition, the degeneracy may be lifted because of the unavoidable static imperfections in the network. We do not expect degeneracy lifting to prevent the observation of non-Abelian effects on pumping, it just imposes a constraint on the duration of the cycle: $T$ should be shorter than $\min \left\{\hbar / \Delta E, \hbar / \tau_{\phi}\right\}$ where $\Delta E$ is the small splitting arising from the non perfect degeneracy of the levels involved and $\tau_{\phi}$ the decoherence time.

This work has been supported by MIUR-PRIN, Institut universitaire de France and EC-Eurosqip. The support of the Lewiner Institute for Theoretical Physics at the Technion is acknowledged.

[1] M. V. Berry, Proc. Roy. Soc. London A392, 45 (1984).

[2] F. Wilczek and A. Zee, Phys. Rev. Lett. 52, 2111 (1984).

[3] B. Simon, Phys. Rev. Lett. 51, 2167 (1983).

[4] Geometric phases in physics, Eds. A. Shapere and F. Wilczek, (World Scientific, Singapore, 1989).

[5] The geometric Phase in quantum systems, A. Bohm et al. Eds., (Springer-Verlag, Berlin, 2003).

[6] D. J. Thouless, Phys. Rev. B 27, 6083 (1983).

[7] M. Büttiker, H. Thomas, and A. Prêtre, Z. Phys. B 94, 133 (1994).

[8] P. W. Brouwer, Phys. Rev. B 58, R10135 (1998).

[9] J. E. Avron et al., Phys. Rev. B 62, R10618 (2000).

[10] H.-Q. Zhou, S. Y. Cho, and R. H. McKenzie, Phys. Rev. Lett. 91, 186803 (2003).

[11] M. Governale et al. Phys. Rev. Lett. 95256801 (2005)

[12] See the analogy with pumping in an Aharonov-Bohm ring, M. Moskalets and M. Büttiker, Phys. Rev. B 68, 075303 (2003); D. Cohen, Physica E 29, 308 (2005).

[13] L. J. Geerligs et al., Z. Phys. B: Condens. Matter 85, 349 (1991).

[14] J.P. Pekola et al., Phys. Rev. B 60, R9931 (1999).

[15] A. O. Niskanen et al., Phys. Rev. Lett. 91, 177003 (2003).

[16] M. Aunola and J. J. Toppari, Phys. Rev. B 68, 020502 (2003).

[17] M. Möttönen et al., Phys. Rev. B 73, 214523 (2006). 
[18] G. Falci et al., Nature (London) 407, 355 (2000).

[19] M. Möttönen, J.J. Vartiainen, and J.P. Pekola, arXiv:0710.5623.

[20] J. Jones et al., Nature (London) 403, 869 (2000).

[21] P. Zanardi and M. Rasetti, Phys. Lett. A 264, 94 (1999).

[22] L. Faoro, J. Siewert, and R. Fazio, Phys. Rev. Lett. 90, 028301 (2003).

[23] A rigorous derivation of Eqs.(4) and (5) from Eq. (1) requires the solution of the Schrödinger equation beyond the adiabatic approximation.

[24] The results presented here admits a straightforward gen- eralization to the case of non-adiabatic cyclic evolutions [Y. Aharonov and A. Anandan, Phys. Rev. Lett. 58, 1593 (1987); A. Anandan, Phys. Lett. A, 133, 171 (1988)].

[25] R.G. Unanyan, B.W. Shore, and K. Bergmann, Phys. Rev. A 59, 2910 (1999).

[26] L.-M. Duan, J. I. Cirac, and P.Zoller, Science 292, 1695 (2001).

[27] J. Siewert, T. Brandes, and G. Falci, Optics Comm. 264, 435 (2006). 